\title{
ON NEUMANN HEMIVARIATIONAL INEQUALITIES
}

\author{
HALIDIAS NIKOLAOS
}

Received 24 October 2001

We derive a nontrivial solution for a Neumann noncoercive hemivariational inequality using the critical point theory for locally Lipschitz functionals. We use the Mountain-Pass theorem due to Chang (1981).

\section{Introduction}

The problem under consideration is a hemivariational inequality of Neumann type. Let $Z \subseteq \mathbb{R}^{N}$ be a bounded domain with a $C^{1}$-boundary $\Gamma$. We have

$$
\begin{array}{ll}
-\operatorname{div}\left(\|D x(z)\|^{p-2} D x(z)\right) \in \partial j_{1}(z, x(z)) & \text { a.e. on } Z, \\
-\frac{\partial x}{\partial n_{p}} \in \partial j_{2}(z, \tau(x(z))) & \text { a.e. on } \Gamma, 2 \leq p<\infty .
\end{array}
$$

Here the boundary condition is in the sense of Kenmochi [7] and $\tau$ is the trace operator (see Kenmochi [7, page 123]).

The study of hemivariational inequalities has been initiated and developed by Panagiotopoulos [8]. Such inequalities arise in physics when we have nonconvex, nonsmooth energy functionals. For applications, one can see [9].

Many authors studied Dirichlet hemivariational inequalities (cf. Gasiński and Papageorgiou [5], Goeleven et al. [6], and others). Here we are interested in finding nontrivial solutions for Neumann hemivariational inequalities.

In Section 2, we recall some facts and definitions from the critical point theory for locally Lipschitz functionals and the subdifferential of Clarke.

\section{Preliminaries}

Let $X$ be a Banach space and let $Y$ be a subset of $X$. A function $f: Y \rightarrow \mathbb{R}$ is said to satisfy a Lipschitz condition (on $Y$ ) provided that, for some nonnegative 
scalar $K$, we have

$$
|f(y)-f(x)| \leq K\|y-x\|
$$

for all points $x, y \in Y$. Let $f$ be a Lipschitz function near a given point $x$, and let $v$ be any other vector in $X$. The generalized directional derivative of $f$ at $x$ in the direction $v$, denoted by $f^{o}(x ; v)$ is defined as follows:

$$
f^{o}(x ; v)=\limsup _{\substack{y \rightarrow x \\ t \downarrow 0}} \frac{f(y+t v)-f(y)}{t},
$$

where $y$ is a vector in $X$ and $t$ a positive scalar. If $f$ is a Lipschitz function of rank $K$ near $x$, then the function $v \rightarrow f^{o}(x ; v)$ is finite, positively homogeneous, subadditive, and satisfies $\left|f^{o}(x ; v)\right| \leq K\|v\|$. In addition, $f^{o}$ satisfies $f^{o}(x ;-v)=$ $(-f)^{o}(x ; v)$. Now we are ready to introduce the generalized gradient which is denoted by $\partial f(x)$ as follows:

$$
\partial f(x)=\left\{w \in X^{*}: f^{o}(x ; v) \geq\langle w, v\rangle \forall v \in X\right\} .
$$

Some basic properties of the generalized gradient of locally Lipschitz functionals are the following:

(a) $\partial f(x)$ is a nonempty, convex, weakly compact subset of $X^{*}$ and $\|w\|_{*} \leq K$ for every $w$ in $\partial f(x)$

(b) for every $v$ in $X$, we have

$$
f^{o}(x ; v)=\max \{\langle w, v\rangle: w \in \partial f(x)\} .
$$

If $f_{1}, f_{2}$ are locally Lipschitz functions then

$$
\partial\left(f_{1}+f_{2}\right) \subseteq \partial f_{1}+\partial f_{2}
$$

Recall the Palais-Smale condition ((PS)-condition) introduced by Chang [2]. Definition 2.1. We say that a Lipschitz function $f$ satisfies the (PS)-condition if for any sequence $\left\{x_{n}\right\},\left|f\left(x_{n}\right)\right|$ is bounded and $\lambda\left(x_{n}\right)=\min _{w \in \partial f\left(x_{n}\right)}\|w\|_{X^{*}} \rightarrow 0$ possesses a convergent subsequence.

The (PS)-condition can also be formulated as follows (see [4]).

$(\mathrm{PS})_{c,+}^{*}$ : whenever $\left(x_{n}\right) \subseteq X,\left(\varepsilon_{n}\right),\left(\delta_{n}\right) \subseteq \mathbb{R}_{+}$are sequences with $\varepsilon_{n} \rightarrow 0, \delta_{n} \rightarrow 0$, and such that

$$
f\left(x_{n}\right) \longrightarrow c, \quad f\left(x_{n}\right) \leq f(x)+\varepsilon_{n}\left\|x-x_{n}\right\| \quad \text { if }\left\|x-x_{n}\right\| \leq \delta_{n},
$$

then $\left(x_{n}\right)$ possesses a convergent subsequence $x_{n^{\prime}} \rightarrow \hat{x}$. 
Similarly, we define the (PS) ${ }_{c}^{*}$ condition from below, (PS) ${ }_{c,-}^{*}$, by interchanging $x$ and $x_{n}$ in inequality (2.6). And finally we say that $f$ satisfies (PS ${ }_{c}^{*}$ provided that it satisfies $(\mathrm{PS})_{c,+}^{*}$ and $(\mathrm{PS})_{c,-\cdot}^{*}$. Note that these two definitions are equivalent when $f$ is a locally Lipschitz functional.

The following theorem is the Mountain-Pass theorem for locally Lipschitz functionals.

THeOREM 2.2. If a locally Lipschitz functional $f: X \rightarrow \mathbb{R}$ on the reflexive Banach space $X$ satisfies the (PS)-condition and the hypotheses:

(i) there exist positive constants $\rho$ and a such that

$$
f(u) \geq a \quad \forall x \in X \text { with }\|x\|=\rho ;
$$

(ii) $f(0)=0$ and there exists a point $e \in X$ such that

$$
\|e\|>\rho, \quad f(e) \leq 0,
$$

then there exists a critical value $c \geq$ a of $f$ determined by

$$
c=\inf _{g \in G} \max _{t \in[0,1]} f(g(t)),
$$

where

$$
G=\{g \in C([0,1], X): g(0)=0, g(1)=e\} .
$$

In what follows, we will use the well-known inequality

$$
\sum_{j=1}^{N}\left(a_{j}(\eta)-a_{j}\left(\eta^{\prime}\right)\right)\left(\eta_{j}-\eta_{j}^{\prime}\right) \geq C\left|\eta-\eta^{\prime}\right|^{p}
$$

for $\eta, \eta^{\prime} \in \mathbb{R}^{N}$, with $a_{j}(\eta)=|\eta|^{p-2} \eta_{j}$.

\section{Existence theorem}

Let $X=W^{1, p}(Z)$. Our hypotheses on $j_{1}, j_{2}$ are the following:

$\mathrm{H}\left(j_{1}\right)$ : the map $j_{1}: Z \times \mathbb{R} \rightarrow \mathbb{R}$ is such that $z \rightarrow j_{1}(z, x)$ is measurable and $x \rightarrow j_{1}(z, x)$ is locally Lipschitz;

(i) for almost all $z \in Z$, all $x \in \mathbb{R}$, and all $v \in \partial j_{1}(z, x)$, we have $|v(z)| \leq$ $c_{1}|x|^{p-1}+c_{2}|x|^{p^{*}-1}$

(ii) there exists $\theta>p$ and $r_{o}>0$ such that for all $|x| \geq r_{o}$, and $v \in \partial j_{1}(z, x)$, we have $0<\theta j_{1}(z, x) \leq v x$, and moreover, there exists some $a \in L^{1}(Z)$ such that $j_{1}(z, x) \geq c_{3}|x|^{\theta}-a(z)$ for every $x \in \mathbb{R}$;

(iii) uniformly for almost all $z \in Z$, we have

$$
\limsup _{x \rightarrow 0} \frac{j_{1}(z, x)}{|x|^{p}} \leq \theta(z)
$$

with $\theta(z) \in L^{\infty}$ and $\theta(z) \leq 0$ with strict inequality in a set of positive measure. 
$\mathrm{H}\left(j_{2}\right)$ : the map $j_{2}(z, x)$ is such that $z \rightarrow j_{2}(z, x)$ is measurable and $j_{2}(z, \cdot)$ is a locally Lipschitz function such that for almost all $z \in Z, x \in \mathbb{R}$, and $v \in \partial j_{2}(z, x)$ we have $|v(z)| \leq \alpha_{1}(z)+c_{1}|x|^{\mu}, 0 \leq \mu<p-1$ with $\alpha_{1} \in L^{\infty}, c_{1}>0, j_{2}(\cdot, 0) \in L^{\infty}(Z)$, and finally $j_{2}(z, \cdot) \geq 0$ for almost all $z \in Z$.

Theorem 3.1. If hypotheses $\mathrm{H}\left(j_{1}\right)$ and $\mathrm{H}\left(j_{2}\right)$ hold, then problem (1.1) has a nontrivial solution $x \in W^{1, p}(Z)$.

Proof. Let $\Phi: W^{1, p}(Z) \rightarrow \mathbb{R}$ and $\psi: W^{1, p}(Z) \rightarrow \mathbb{R}_{+}$be defined by

$$
\Phi(x)=-\int_{Z} j_{1}(z, x(z)) d z, \quad \psi(x)=\frac{1}{p}\|D x\|_{p}^{p}+\int_{\Gamma} j_{2}(z, \tau(x(z))) d \sigma .
$$

Clearly, $\Phi$ is locally Lipschitz (see Chang [2]), while we can check that $\psi$ is locally Lipschitz too. Set $R=\Phi+\psi$.

Claim 3.2. The function $R(\cdot)$ satisfies the (PS)-condition (in the sense of Costa and Gonçalves).

We start with $(\mathrm{PS})_{c,+}$ first. Let $\left\{x_{n}\right\}_{n \geq 1} \subseteq W^{1, p}(Z)$ such that $R\left(x_{n}\right) \rightarrow c$ when $n \rightarrow \infty$ and

$$
R\left(x_{n}\right) \leq R(x)+\varepsilon_{n}\left\|x-x_{n}\right\| \quad \text { with } \quad\left\|x-x_{n}\right\| \leq \delta_{n} .
$$

The above inequality is equivalent to the following:

$$
R(x)-R\left(x_{n}\right) \geq-\varepsilon_{n}\left\|x-x_{n}\right\| \quad \text { with }\left\|x-x_{n}\right\| \leq \delta_{n}
$$

where $\varepsilon_{n}, \delta_{n} \rightarrow 0$. Choose $x=x_{n}+\delta x_{n}$ with $\delta\left\|x_{n}\right\| \leq \delta_{n}$. Divide by $\delta$. So, if $\delta \rightarrow 0$ we have

$$
\lim _{\delta \rightarrow 0} \frac{R\left(x_{n}+\delta x_{n}\right)-R\left(x_{n}\right)}{\delta} \leq R^{o}\left(x_{n} ; x_{n}\right) .
$$

Then we obtain

$$
R^{o}\left(x_{n} ; x_{n}\right) \geq-\varepsilon_{n}\left\|x_{n}\right\| .
$$

For the (PS) $)_{c,-}$ we have the following: let $\left\{x_{n}\right\}_{n \geq 1} \subseteq W^{1, p}(Z)$ such that $R\left(x_{n}\right) \rightarrow$ $c$ when $n \rightarrow \infty$ and

$$
R(x) \leq R\left(x_{n}\right)+\varepsilon_{n}\left\|x-x_{n}\right\| \quad \text { with } \quad\left\|x-x_{n}\right\| \leq \delta_{n} .
$$

The above inequality is equivalent to the following:

$$
0 \leq(-R)(x)-(-R)\left(x_{n}\right)+\varepsilon_{n}\left\|x-x_{n}\right\| \text { with }\left\|x-x_{n}\right\| \leq \delta_{n} .
$$

Choose here $x=x_{n}-\delta x_{n}$ with $\delta\left\|x_{n}\right\| \leq \delta_{n}$. We obtain

$$
0 \leq(-R)\left(x_{n}+\delta\left(-x_{n}\right)\right)-(-R)\left(x_{n}\right)+\varepsilon_{n} \delta\left\|x_{n}\right\| .
$$


Divide this by $\delta$. In the limit, we have

$$
0 \leq \lim _{\delta \rightarrow 0} \frac{(-R)\left(x_{n}+\delta\left(-x_{n}\right)\right)-(-R)\left(x_{n}\right)}{\delta}+\varepsilon_{n}\left\|x_{n}\right\| .
$$

Note that

$$
\lim _{\delta \rightarrow 0} \frac{(-R)\left(x_{n}+\delta\left(-x_{n}\right)\right)-(-R)\left(x_{n}\right)}{\delta} \leq(-R)^{o}\left(x_{n} ;-x_{n}\right)=R^{o}\left(x_{n} ; x_{n}\right) .
$$

So finally we obtain again (3.6).

Also,

$$
\frac{1}{p}\left\|D\left(x_{n}+\delta x_{n}\right)\right\|_{p}^{p}-\frac{1}{p}\left\|D x_{n}\right\|=-\frac{1}{p}\left\|D x_{n}\right\|_{p}^{p}\left(1-(1+\delta)^{p}\right) .
$$

So if we divide this by $\delta$ and let $\delta \rightarrow 0$, we have that it is equal to $\left\|D x_{n}\right\|_{p}^{p}$. Finally, there exists $v_{n}(z) \in \partial \Phi\left(x_{n}\right)$ such that $\left\langle v_{n}, x_{n}\right\rangle=\Phi^{o}\left(x_{n} ; x_{n}\right)$ and $w_{n} \in$ $\partial j_{2}\left(z, \tau\left(x_{n}(z)\right)\right)$ such that

$$
\left\langle w_{n}, x_{n}\right\rangle_{\Gamma}=\psi_{1}^{o}\left(x_{n} ; x_{n}\right) \quad \text { with } \psi_{1}(x)=\int_{\Gamma} j_{2}(z, \tau(x(z))) d \sigma .
$$

Note that

$$
v_{n} \in \partial\left(-\int_{Z} j_{1}\left(z, x_{n}(z)\right) d z\right)=-\partial \int_{Z} j_{1}\left(z, x_{n}(z)\right) d z
$$

So, from (3.6), it follows that

$$
\int_{Z} v_{n} x_{n}(z) d z-\left\|D x_{n}\right\|_{p}^{p}-\int_{\Gamma} w_{n} x_{n} d \sigma \leq \varepsilon_{n}\left\|x_{n}\right\|,
$$

for some $v_{n} \in \partial\left(\int_{Z} j_{1}\left(z, x_{n}(z)\right) d z\right)$.

Suppose that $\left\{x_{n}\right\} \subseteq W^{1, p}(Z)$ was unbounded. Then (at least for a subsequence), we may assume that $\left\|x_{n}\right\| \rightarrow \infty$. Let $y_{n}=x_{n} /\left(\left\|x_{n}\right\|\right), n \geq 1$, and it is easy to see that $\left\|y_{n}\right\|=1$. By passing to a subsequence if necessary, we may assume that

$$
\begin{array}{cl}
y_{n} \stackrel{w}{\longrightarrow} y & \text { in } W^{1, p}(Z), \\
y_{n} \longrightarrow y & \text { in } L^{p}(Z), \\
y_{n}(z) \longrightarrow y(z) & \text { a.e. on } Z \text { as } n \longrightarrow \infty, \\
\left|y_{n}(z)\right| \leq k(z) & \text { a.e. on } Z \text { with } k \in L^{p}(Z) .
\end{array}
$$

Recall that from the choice of the sequence $\left\{x_{n}\right\}$, we have $\left|R\left(x_{n}\right)\right| \leq M_{1}$ for some $M_{1}>0$ and all $n \geq 1$,

$$
\frac{\theta}{p}\left\|D x_{n}\right\|_{p}^{p}+\theta \int_{\Gamma} j_{2}\left(z, \tau\left(x_{n}(z)\right)\right) d \sigma-\theta \int_{Z} j_{1}\left(z, x_{n}(z)\right) d z \leq \theta M_{1} .
$$


On the other hand, we have

$$
\int_{Z} v_{n} x_{n}(z) d z-\left\|D x_{n}\right\|_{p}^{p}-\int_{\Gamma} w_{n} x_{n} d \sigma \leq \varepsilon_{n}\left\|x_{n}\right\| .
$$

Adding inequalities (3.17) and (3.18) we obtain

$$
\begin{gathered}
\left(\frac{\theta}{p}-1\right)\left\|D x_{n}\right\|_{p}^{p}+\int_{Z}\left(v_{n}(z) x_{n}(z)-\theta j_{1}\left(z, x_{n}(z)\right)\right) d z \\
+\int_{\Gamma}\left(j_{2}\left(z, \tau\left(x_{n}(z)\right)\right)-w_{n}(z) x_{n}(z)\right) d \sigma \\
\leq \theta M_{1}+\varepsilon_{n}\left\|x_{n}\right\| .
\end{gathered}
$$

From $\mathrm{H}\left(j_{1}\right)$ (ii), we have that for all $|x|>M$ and $v \in \partial j_{1}(z, x), 0<\theta j_{1}(z, x) \leq$ $v x$. From $\mathrm{H}\left(j_{1}\right)(i)$, we know that $|v| \leq c_{1}|x|^{p-1}+c_{2}|x|^{p^{*}-1}$. Using Lebourg mean value theorem (see Clarke [3, Theorem 2.3.7, page 41]) we have that for all $x \in \mathbb{R}$

$$
j_{1}(z, x)-j_{1}(z, 0)=w x
$$

with $w \in \partial j_{1}(z, s)$ where $s \in(0, x)$. Recall that $j_{1}(z, 0) \in L^{\infty}(Z)$. So

$$
\left|j_{1}(z, x)\right| \leq c_{1}+c_{2}|x|^{p}+c_{3}|x|^{p^{*}} .
$$

So for $|x| \leq M$, we have that $|v| \leq C$ and $\left|j_{1}(z, x)\right| \leq C$ for all $v \in \partial j_{1}(z, x)$ for some $C>0$. Thus, there exists some $M>0$ such that $v x-\theta j_{1}(z, x)+M \geq 0$ for all $x \in \mathbb{R}$.

Therefore, (3.19) becomes

$$
\left(\frac{\theta}{p}-1\right)\left\|D x_{n}\right\|_{p}^{p}+\int_{\Gamma}\left(j_{2}\left(z, \tau\left(x_{n}(z)\right)\right)-w_{n}(z) x_{n}(z)\right) d \sigma \leq \theta M_{1}+\varepsilon_{n}\left\|x_{n}\right\|+M .
$$

Dividing by $\left\|x_{n}\right\|^{p}$, we get

$$
\frac{\int_{\Gamma}\left(j_{2}\left(z, \tau\left(x_{n}(z)\right)\right)-w_{n}(z) x_{n}(z)\right) d \sigma}{\left\|x_{n}\right\|^{p}} \longrightarrow 0 .
$$

Indeed, from the Lebourg mean value theorem, we have that for any $x \in \mathbb{R}$,

$$
j_{2}(z, x)-j_{2}(z, 0)=w x,
$$

with $w \in \partial j_{2}(z, s)$ where $s \in(0, x)$. From $\mathrm{H}\left(j_{2}\right)$, we have that for every $w \in$ $\partial j_{2}(z, s),|w| \leq c_{1}+c_{2}|s|^{\mu}$. Moreover, note that $j_{2}(z, 0) \in L^{\infty}$. So,

$$
\left|j_{2}(z, x)\right| \leq c_{1}|x|+c_{2}|x|^{\mu+1}+c_{3} .
$$


Thus,

$$
\begin{aligned}
& \frac{\int_{\Gamma}\left(j_{2}\left(z, \tau\left(x_{n}(z)\right)\right)-w_{n}(z) x_{n}(z)\right) d \sigma}{\left\|x_{n}\right\|^{p}} \\
& \quad \leq \int_{\Gamma} \frac{c_{1}\left|x_{n}(z)\right|}{\left\|x_{n}\right\|^{p}} d \sigma+\int_{\Gamma} \frac{c_{2}\left|x_{n}(z)\right|^{\mu+1}}{\left\|x_{n}\right\|^{p}} d \sigma+\frac{c_{4}}{\left\|x_{n}\right\|^{p}} \\
& \quad \leq c_{1} \frac{\left\|x_{n}\right\|_{L^{1}(\Gamma)}}{\left\|x_{n}\right\|^{p}}+c_{2} \frac{\left\|x_{n}\right\|_{L^{\mu+1}(\Gamma)}^{\mu+1}}{\left\|x_{n}\right\|^{p}}+\frac{c_{4}}{\left\|x_{n}\right\|^{p}} .
\end{aligned}
$$

Note that

$$
\begin{gathered}
\left\|x_{n}\right\|_{L^{1}(\Gamma)} \leq K\left\|x_{n}\right\|_{1 / q, 1, \Gamma} \leq C\left\|x_{n}\right\|_{1, p, Z}, \\
\left\|x_{n}\right\|_{L^{\mu+1}(\Gamma)}^{\mu+1} \leq K\left\|x_{n}\right\|_{1 / q, p, \Gamma}^{\mu+1} \leq C\left\|x_{n}\right\|_{1, p, Z}^{\mu+1}
\end{gathered}
$$

(see Adams [1, page 217]), recall that $\mu+1<p$. Now we have finished the claim.

Going back to (3.22) we have that $\left\|D y_{n}\right\| \rightarrow 0$. From the weak lower semicontinuity of the norm functional, we have that $\|D y\| \leq \liminf \left\|D y_{n}\right\| \leq \limsup \left\|D y_{n}\right\|$ $\rightarrow 0$. Therefore, we infer that $y_{n} \rightarrow y$ in $W^{1, p}(Z)$ (recall that $y_{n} \rightarrow y$ weakly in $W^{1, p}(Z)$ and $\left.\left\|D y_{n}\right\| \rightarrow\|D y\|=0\right)$. So, $y=\xi \in \mathbb{R}$. But, $\left\|y_{n}\right\|=1$, so $\|y\|=1$, thus $y=\xi \neq 0$. Suppose that $\xi>0$, then $x_{n}(z) \rightarrow \infty$. From $\mathrm{H}\left(j_{1}\right)$ (ii) we have that, for all $x \in \mathbb{R}, j_{1}(z, x) \geq c_{1}|x|^{\theta}-a(z)$. So it is clear that

$$
\begin{aligned}
\frac{R\left(x_{n}\right)}{\left\|x_{n}\right\|^{\theta}} \leq & \frac{1}{p}\left\|D y_{n}\right\|_{p}^{p} \frac{1}{\left\|x_{n}\right\|^{\theta-p}}-c_{1} \int_{Z}\left|y_{n}(z)\right|^{\theta} d z \\
& +\int_{Z} \frac{a(z)}{\left\|x_{n}\right\|^{\theta}} d z+\frac{\int_{\Gamma} j_{2}\left(z, \tau\left(x_{n}(z)\right)\right) d \sigma}{\left\|x_{n}\right\|^{\theta}} .
\end{aligned}
$$

Recall that from the choice of the sequence we have that

$$
\frac{R\left(x_{n}\right)}{\left\|x_{n}\right\|^{\theta}} \geq-\frac{M_{1}}{\left\|x_{n}\right\|^{\theta}} \text {. }
$$

As before it is easy to see that

$$
\frac{\int_{\Gamma} j_{2}\left(z, \tau\left(x_{n}(z)\right)\right) d \sigma}{\left\|x_{n}\right\|^{\theta}} \longrightarrow 0, \quad \frac{1}{p}\left\|D y_{n}\right\|_{p}^{p} \frac{1}{\left\|x_{n}\right\|^{\theta-p}} \longrightarrow 0 .
$$

So from (3.28) we have that $0 \leq-c_{1}|\xi|^{\theta}|Z|$. But this is a contradiction. So $\left\{x_{n}\right\} \subseteq W^{1, p}(Z)$ is bounded.

From the properties of the subdifferential of Clarke, we have

$$
\begin{aligned}
\partial R\left(x_{n}\right) & \subseteq \partial \Phi\left(x_{n}\right)+\partial \psi\left(x_{n}\right) \\
& \subseteq \partial \Phi\left(x_{n}\right)+\partial\left(\frac{1}{p}\left\|D x_{n}\right\|_{p}^{p}\right)+\int_{\Gamma} \partial j_{2}\left(z, \tau\left(x_{n}(z)\right)\right) d \sigma
\end{aligned}
$$


(see Clarke [3, page 83]). So we have

$$
\left\langle w_{n}, y\right\rangle=\left\langle A x_{n}, y\right\rangle+\left\langle\tau\left(r_{n}\right), y\right\rangle_{\Gamma}-\int_{Z} v_{n}(z) y(z) d z
$$

with $r_{n}(z) \in \partial j_{2}\left(z, \tau\left(x_{n}(z)\right)\right), v_{n}(z) \in \partial j_{1}\left(z, x_{n}(z)\right)$, and $w_{n}$ the element with minimal norm of the subdifferential of $\mathbb{R}$ and $A: W^{1, p}(Z) \rightarrow W^{1, p}(Z)^{*}$ such that $\langle A x, y\rangle=\int_{Z}\left(\|D x(z)\|^{p-2}(D x(z), D y(z))_{\mathbb{R}^{N}}\right) d z$. But $x_{n} \stackrel{w}{\rightarrow} x$ in $W^{1, p}(Z)$, so $x_{n} \rightarrow$ $x$ in $L^{p}(Z)$ and $x_{n}(z) \rightarrow x(z)$ a.e. on $Z$ by virtue of the compact embedding $W^{1, p}(Z) \subseteq L^{p}(Z)$.

Note that the trace of $x_{n}$ belongs to $W^{1 / q, p}(\Gamma)$, thus, from $\mathrm{H}\left(j_{2}\right)$, the trace of $r_{n} \in L^{q}(\Gamma)$. Recall that there exists some $K>0$ such that $\left\|x_{n}\right\|_{1 / q, p, \Gamma} \leq K\left\|x_{n}\right\|_{1, p, Z}$. Therefore, $r_{n}$ is bounded in $L^{q}(\Gamma)$ and moreover, in $\left(W^{1 / q, p}(\Gamma)\right)^{*}$ (the dual space of $\left.W^{1 / q, p}(\Gamma)\right)$. Choose $y=x_{n}-x$, then we obtain

$$
\left|\left\langle\tau\left(r_{n}\right), x_{n}-x\right\rangle_{\Gamma}\right| \longrightarrow 0
$$

With $\langle\cdot, \cdot\rangle_{\Gamma}$ we denote the natural pairing of $\left(W^{1 / q, p}(\Gamma),\left(W^{1 / q, p}(\Gamma)\right)^{*}\right)$.

Then in the limit we have that $\lim \sup \left\langle A x_{n}, x_{n}-x\right\rangle=0$ (note that $v_{n}$ is bounded in $\left.L^{p^{*}}(Z)\right)$. By virtue of inequality (2.11), we have that $D x_{n} \rightarrow D x$ in $L^{p}(Z)$. So we have $x_{n} \rightarrow x$ in $W^{1, p}(Z)$. The claim is proved.

For every $\xi \in \mathbb{R}, \xi \neq 0$, we have

$$
\begin{aligned}
R(\xi) & =\int_{\Gamma} j_{2}(z, \xi) d \sigma-\int_{Z} j_{1}(z, \xi) d z \Longrightarrow \frac{1}{|\xi|^{\theta}} R(\xi) \\
& \leq \frac{1}{|\xi|^{\theta}} \int_{\Gamma} j_{2}(z, \xi) d \sigma-\frac{1}{|\xi|^{\theta}} \int_{Z} j_{1}(z, \xi) d z
\end{aligned}
$$

As before we show that

$$
-\frac{1}{|\xi|^{\theta}} \int_{Z} j_{1}(z, \xi) d z \leq-c_{1} \frac{|\xi|^{\theta}}{|\xi|^{\theta}}, \quad \frac{1}{|\xi|^{\theta}} \int_{\Gamma} j_{2}(z, \xi) d \sigma \longrightarrow 0
$$

Thus $R(\xi) \rightarrow-\infty$ as $|\xi| \rightarrow \infty$.

In order to use the Mountain-Pass theorem, it remains to show that there exists $\rho>0$ such that for $\|x\|=\rho, R(x) \geq a>0$. In fact, we will show that for every sequence $\left\{x_{n}\right\} \subseteq W^{1, p}(Z)$ with $\left\|x_{n}\right\|=\rho_{n} \downarrow 0, R\left(x_{n}\right)>0$. Indeed, suppose not. Then there exists some sequence $\left\{x_{n}\right\}$ such that $R\left(x_{n}\right) \leq 0$. Thus,

$$
\frac{1}{p}\left\|D x_{n}\right\|_{p}^{p} \leq \int_{Z} j_{1}\left(z, x_{n}(z)\right) d z
$$

recall that $j_{2} \geq 0$. Dividing this inequality by $\left\|x_{n}\right\|^{p}$ and letting $y_{n}(z)=x_{n}(z) /\left\|x_{n}\right\|$, then

$$
\left\|D y_{n}\right\|_{p}^{p} \leq \int_{Z} p \frac{j_{1}\left(z, x_{n}(z)\right)}{\left\|x_{n}\right\|^{p}} d z
$$


From $\mathrm{H}\left(j_{1}\right)$ (iii) we have that for almost all $z \in Z$, for any $\varepsilon>0$, we can find $\delta>0$ such that for $|x| \leq \delta$,

$$
p j_{1}(z, x) \leq(\theta(z)+\varepsilon)|x|^{p} .
$$

On the other hand, as before for almost all $z \in Z$ and all $|x| \geq \delta$, we have

$$
p\left|j_{1}(z, x)\right| \leq c_{1}|x|^{p}+c_{2}|x|^{p^{*}} .
$$

Thus we can always find $\gamma>0$ such that $p\left|j_{1}(z, x)\right| \leq(\theta(z)+\varepsilon)|x|^{p}+\gamma|x|^{p^{*}}$ for all $x \in \mathbb{R}$. Indeed, choose $\gamma \geq c_{2}+\left|\theta(z)+\varepsilon-c_{1}\right||\delta|^{p-p^{*}}$, we obtain

$$
\begin{aligned}
\left\|D y_{n}\right\|_{p}^{p} & \leq \int_{Z}(\theta(z)+\varepsilon)\left|y_{n}(z)\right|^{p} d z+\gamma \int_{Z} \frac{\left|x_{n}(z)\right|^{p^{*}}}{\left\|x_{n}\right\|^{p}} d z \\
& \leq \int_{Z}(\theta(z)+\varepsilon)\left|y_{n}(z)\right|^{p} d z+\gamma_{1}\left\|x_{n}\right\|^{p^{*}-p} .
\end{aligned}
$$

Here we have used the fact that $W^{1, p}(Z)$ embeds continuously in $L^{p^{*}}(Z)$. So

$$
0 \leq\left\|D y_{n}\right\|_{p}^{p} \leq \varepsilon\left\|y_{n}\right\|_{p}^{p}+\gamma_{1}\left\|x_{n}\right\|^{p^{*}-p} .
$$

Therefore, in the limit we have that $\left\|D y_{n}\right\|_{p} \rightarrow 0$. Recall that $y_{n} \rightarrow y$ weakly in $W^{1, p}(Z)$. So $\|D y\|_{p} \leq \liminf \left\|D y_{n}\right\|_{p} \leq \limsup \left\|D y_{n}\right\|_{p} \rightarrow 0$. So $\|D y\|_{p}=0$, thus $y=\xi \in \mathbb{R}$. Note that $D y_{n} \rightarrow D y$ weakly in $L^{p}(Z)$ and $\left\|D y_{n}\right\|_{p} \rightarrow\|D y\|_{p}$ so $y_{n} \rightarrow y$ in $W^{1, p}(Z)$. Since $\left\|y_{n}\right\|=1,\|y\|=1$ so $\xi \neq 0$. Suppose that $\xi>0$. Going back to (3.40), we have

$$
0 \leq \int_{Z}(\theta(z)+\varepsilon) y_{n}^{p}(z) d z+\gamma_{1}\left\|x_{n}\right\|^{p^{*}-p}
$$

In the limit we have

$$
0 \leq \int_{Z}(\theta(z)+\varepsilon) \xi^{p} d z \leq \varepsilon \xi^{p}|Z|
$$

recall that $\theta(z) \leq 0$. Thus $\int_{Z} \theta(z) \xi^{p} d z=0$. But this is a contradiction. So the claim is proved.

By Theorem 2.2, there exists $x \in W^{1, p}(Z)$ such that $0 \in \partial R(x)$. That is, $0 \in$ $\partial \Phi(x)+\partial \psi(x)$. So, we can say that

$$
\int_{Z} w(z) y(z)=\int_{Z}\|D x(z)\|^{p-2}(D x(z), D y(z)) d z+\int_{\Gamma} v(z) x(z) d \sigma
$$

for some $w \in L^{q^{*}}(Z)$ such that $w(z) \in \partial j_{1}(z, x(z))$ for some $v \in \partial j_{2}(z, \tau(x(z)))$ and for every $y \in W^{1, p}(Z)$. Choose now $y=s \in C_{o}^{\infty}(Z)$, we obtain

$$
\int_{Z} w(z) s(z)=\int_{Z}\|D x(z)\|^{p-2}(D x(z), D s(z)) d z .
$$




\section{On Neumann hemivariational inequalities}

But $\operatorname{div}\left(\|D x(z)\|^{p-2} D x(z)\right) \in L^{q^{*}}(Z)$ because $w \in L^{q^{*}}(Z)$ (see Kenmochi [7, Proposition 3.1, page 132]).

Going back to (3.44) and letting $y=C^{\infty}(Z)$ and finally using [7, the Green formula 1.6], we have that $-\partial x / \partial n_{p} \in \partial j_{2}(z, \tau(x(z)))$.

\section{References}

[1] R. A. Adams, Sobolev Spaces, Pure and Applied Mathematics, vol. 65, Academic Press, New York, 1975.

[2] K. C. Chang, Variational methods for nondifferentiable functionals and their applications to partial differential equations, J. Math. Anal. Appl. 80 (1981), no. 1, 102129.

[3] F. H. Clarke, Optimization and Nonsmooth Analysis, Canadian Mathematical Society Series of Monographs and Advanced Texts, John Wiley \& Sons, New York, 1983.

[4] D. G. Costa and J. V. A. Gonçalves, Critical point theory for nondifferentiable functionals and applications, J. Math. Anal. Appl. 153 (1990), no. 2, 470-485.

[5] L. Gasiński and N. S. Papageorgiou, Nonlinear hemivariational inequalities at resonance, J. Math. Anal. Appl. 244 (2000), no. 1, 200-213.

[6] D. Goeleven, D. Motreanu, and P. D. Panagiotopoulos, Multiple solutions for a class of eigenvalue problems in hemivariational inequalities, Nonlinear Anal. 29 (1997), no. 1, 9-26.

[7] N. Kenmochi, Pseudomonotone operators and nonlinear elliptic boundary value problems, J. Math. Soc. Japan 27 (1975), 121-149.

[8] P. D. Panagiotopoulos, Hemivariational inequalities and their applications, Topics in Nonsmooth Mechanics, Birkhäuser, Basel, 1988, pp. 75-142.

[9] - Hemivariational Inequalities. Applications in Mechanics and Engineering, vol. 16, Springer-Verlag, Berlin, 1993.

Halidias Nikolaos: Department of Statistics and Actuarial Science, University of the Aegean, Karlovassi, 83200, Samos, Greece

E-mail address: nick@aegean.gr 


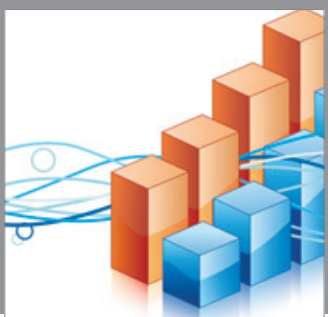

Advances in

Operations Research

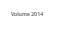

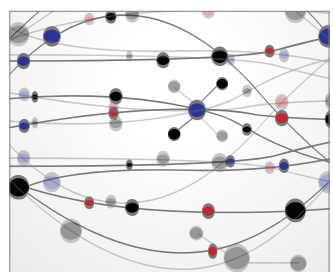

\section{The Scientific} World Journal
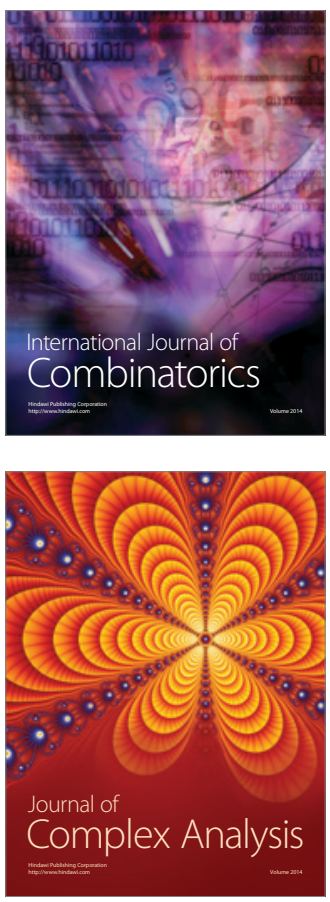

International Journal of

Mathematics and

Mathematical

Sciences
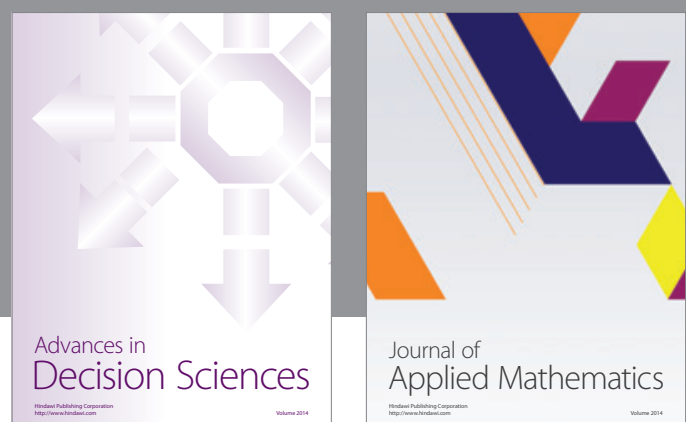

Journal of

Applied Mathematics
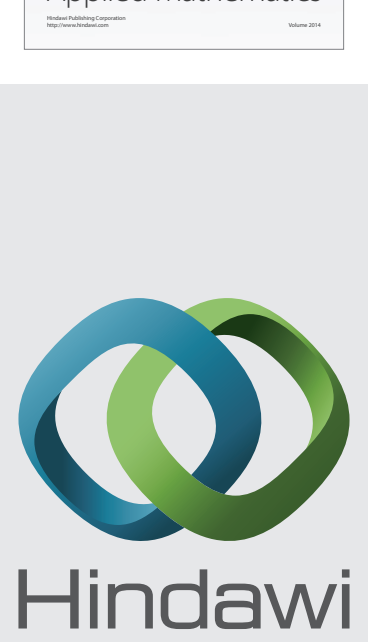

Submit your manuscripts at http://www.hindawi.com
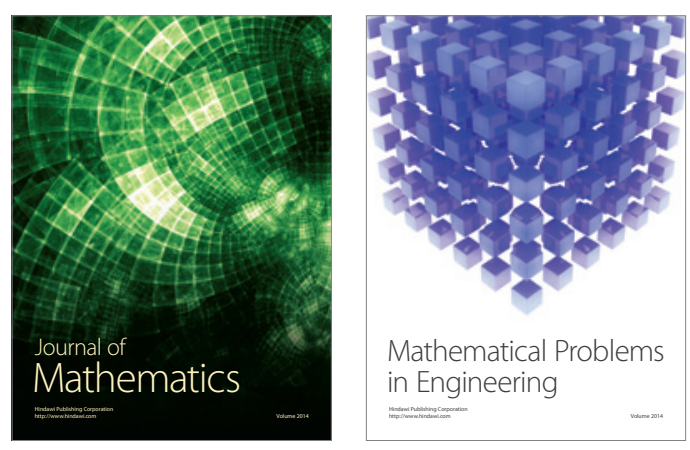

Mathematical Problems in Engineering
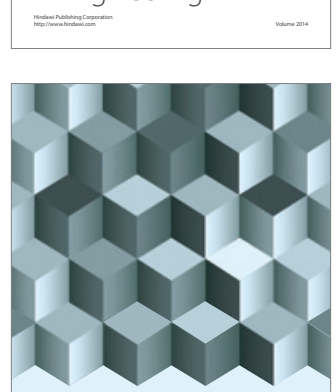

Journal of

Function Spaces
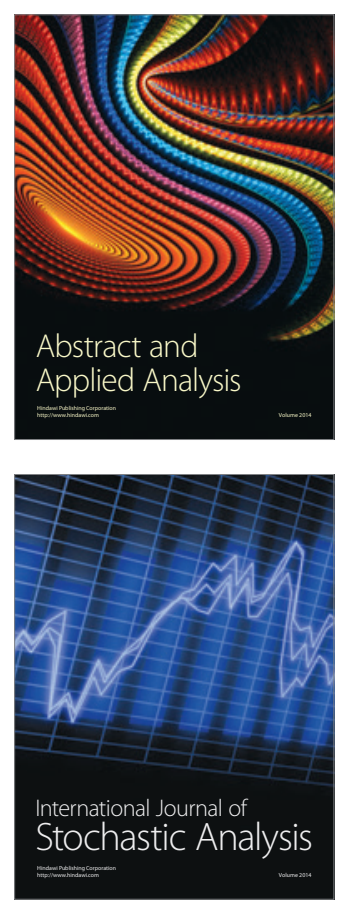

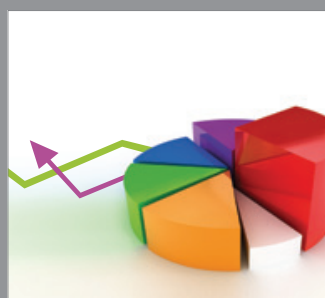

ournal of

Probability and Statistics

Promensencen
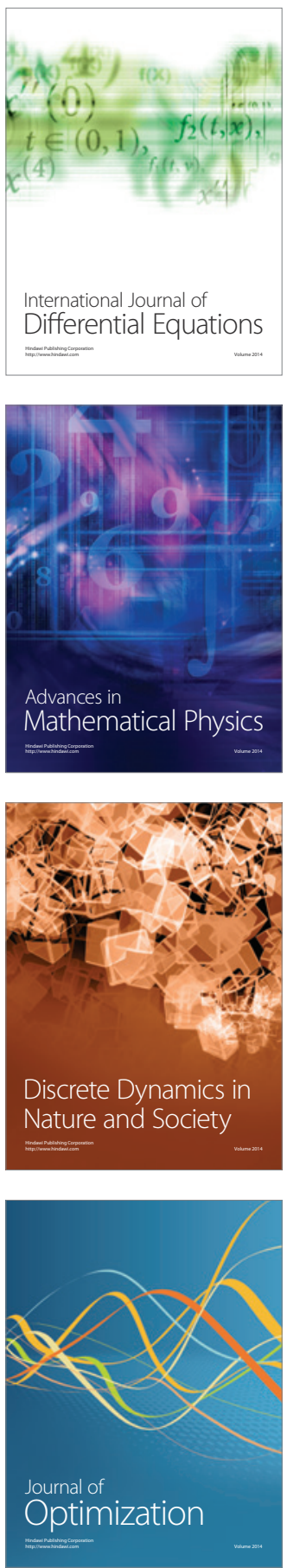